\title{
V-pi Reduction by Using Modulation Index Booster (MiBo) in RF Links
}

\author{
David Borlaug ${ }^{1}$, Rasul Torun ${ }^{2}$, Ozdal Boyraz ${ }^{2}$, and Bahram Jalali ${ }^{1}$ \\ ${ }^{1}$ Department of Electrical Engineering, University of California, Los Angeles, California 90095, USA \\ ${ }^{2}$ Department of Electrical Engineering, University of California, Irvine, California 92617, USA \\ Author e-mail address: jalali@ucla.edu
}

Abstract: The spur-free dynamic range (SFDR) of a modulation index booster (MiBo) link is experimentally and numerically demonstrated. $14.2 \mathrm{~dB}$ RF gain is demonstrated for 5 -fold $V_{\pi}$ reduction at $10 \mathrm{GHz}$ with an $\mathrm{SFDR}_{3}$ better than $90 \mathrm{~dB} / \mathrm{Hz}$. OCIS codes: (060.2330) Fiber optics communications; (060.4080) Modulation; (190.0190) Nonlinear optics.

\section{Introduction}

The electrooptic (EO) modulator is the key mixing component that translates electronic domain information to the optical domain. Conventional electrooptic modulator performance is frustrated by the tradeoffs between low bias voltages, high-frequency operation and bias drift across the operational bandwidth. In the modulation index booster (MiBo), an electro-optic modulator generates modulation sidebands, which then stimulate sideband amplification through modulation instability (MI) in a third-order nonlinear optical material, such as fibers or planar waveguides. The approach reduces the required drive voltage by amplifying the modulation sideband at the expense of the carrier, thereby lowering the effective half-wave voltage $V_{\pi}$ dramatically. Modulation instability is a ubiquitous nonlinear process that arises in diverse contexts such as hydrodynamics, free electron lasers, traffic queues, optical rogue waves and high gain parametric amplification. The use of MI in MiBo paves the way for positive link gain and better link performance for low loss RF links pertinent to radar and analog communications.

The MiBo approach was first demonstrated numerically using ideal optical and electronic component models [1]. Numerical results of MiBo has been verified experimentally, using a swept RF input tone and optical spectrum analyzer [2]. The approach demonstrated a 10 -fold $V_{\pi}$ reduction without requiring a costly high-speed photodiode or sampling oscilloscope. In this paper, the spur-free dynamic range (SFDR) of the MiBo approach is experimentally and numerically measured. Experimental SFDR data is reported at $10 \mathrm{GHz}$. By utilizing commercial link simulators (VPI transmission maker) with realistic component models simulations at $10 \mathrm{GHz}$ and $100 \mathrm{GHz}$ enable predictive SFDR analysis.

\section{Principle and Numerical Setup}

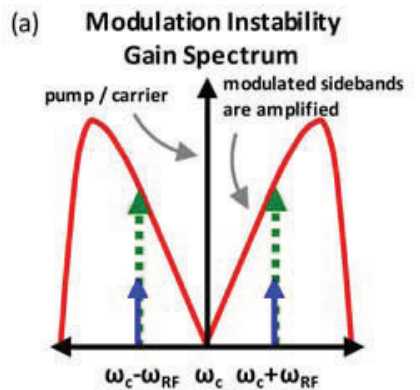

(b) Modulation Depth

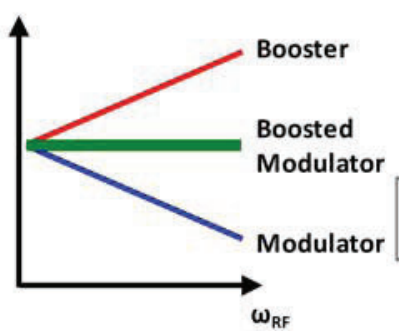

(c)

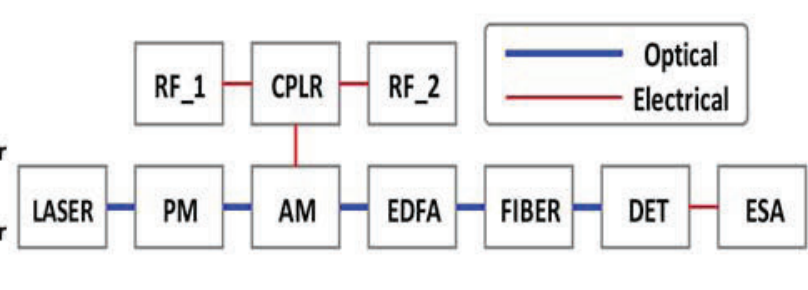

Fig. 1(a) (color online) Principle of electrooptic modulation enhancement using modulation instability boosting (MiBo). Modulation sidebands grow due to MI. Note that the gain increases with RF frequency. 1(b) Diagram of modulation depth vs. RF frequency. A low-voltage highfrequency modulator is achieved as the booster inherently compensates the high-frequency roll-off of the modulator. 1(c) Numerical setup. LASER; PM: phase modulator; AM: amplitude modulator; CPLR: RF coupler; RF_[\#]: RF synthesizer, numeric designator; EDFA: erbium doped fiber amplifier; FIBER; DET: photodetector; ESA: electrical spectrum analyzer.

MI is a parametric process that can amplify RF sidebands at the expense of the carrier frequency, Fig 1a. The gain bandwidth and gain shape is determined by the dispersion and nonlinearity of the waveguide and the peak power (also called pump) of the carrier frequency, formulated as $g\left(f_{R F}\right)=\left|\beta_{2} f_{R F}\right|\left[\left(4 \gamma P /\left|\beta_{2}\right|\right)-f_{R F}^{2}\right]^{1 / 2}$. The peak gain occurs at frequency separation of $\Delta f_{\text {peak }}=\sqrt{2 \gamma P /\left|\beta_{2}\right|}$ and then rolls off, [3]. Here, $\beta_{2}$ is the fiber's second-order dispersion coefficient, $\gamma$ is the Kerr nonlinear coefficient, $P$ is the optical power, and $f_{R F}$ is the radio frequency (RF). The gain shape can be adjusted to compensate the EO modulator's roll-off for low-voltage high-frequency modulation or to achieve net link gain by choosing the correct combination of the above coefficients, as depicted in fig. 1(b). 
The experimental and simulated setup are shown in fig. 1(c). Simulated components are described by VPI transmission maker's component library which is based on commercially available off the shelf components. The simulated laser is a $1550 \mathrm{~nm}$ DFB laser producing $5 \mathrm{~mW}$ output power with a RIN of $-165 \mathrm{~dB} / \mathrm{Hz}$. Simulated laser output is amplified to $235 \mathrm{~mW}$ with an EDFA that exhibits a $6 \mathrm{~dB}$ noise figure. The simulated amplitude modulator has $4.1 \mathrm{~V} V_{\pi}$ voltage and the $-3.0 \mathrm{~dB}$ point of the $\left|S_{21}(f)\right|$ data is set to be at $15 \mathrm{GHz}$. The insertion loss of the modulator is set to be $5.5 \mathrm{~dB}$. The $2 \mathrm{~km}$ highly nonlinear fiber is modeled using the nonlinear Schrodinger equation [3] with the following coefficients: $\alpha=1 \mathrm{~dB} / \mathrm{km}, \beta_{2}=-10.9 \mathrm{ps}^{2} / \mathrm{km}, \beta_{3}=0.062 \mathrm{ps}^{3} / \mathrm{km}$, and $\gamma=11.6 \mathrm{~W}^{-1} \mathrm{~km}^{-1}$. Simulations use a $15 \mathrm{GHz}$ photodetector with $0.70 \mathrm{~A} / \mathrm{W}$ responsivity and set the optical power input at the detector to $+13 \mathrm{dBm}$. We use ideal RF synthesizers, RF couplers, ESA and other ideal components.

\section{Results and Discussion}

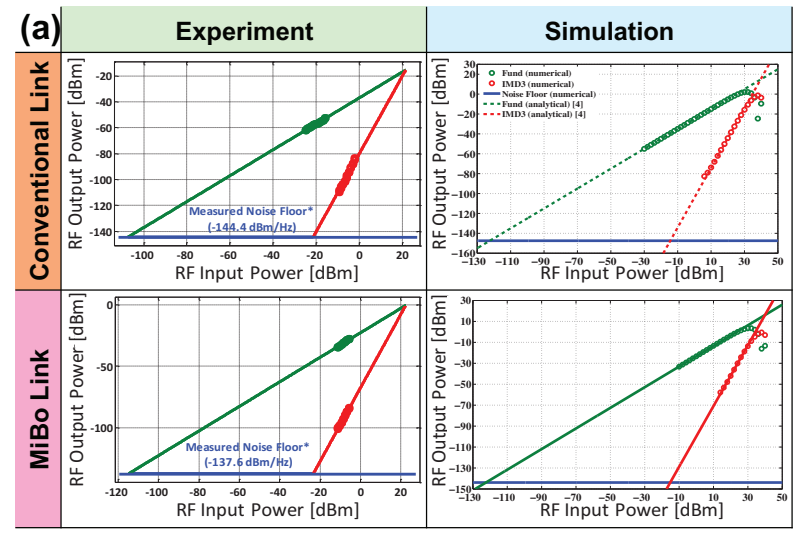

\begin{tabular}{|c|c|c|c|c|}
\cline { 2 - 5 } & \multicolumn{2}{|c|}{ (b) } & \multicolumn{2}{c|}{ Simulation } \\
\cline { 2 - 5 } \multicolumn{1}{c|}{} & Conventional & MiBo & Conventional & MiBo \\
\hline $\begin{array}{c}\text { IIP3 } \\
{[\mathrm{dBm}]}\end{array}$ & 21.5 & 22.5 & 39.7 & 39.92 \\
\hline $\begin{array}{c}\text { OIP3 } \\
{[\mathrm{dBm}]}\end{array}$ & -15.5 & -0.3 & 14.84 & 16.05 \\
\hline $\begin{array}{c}\text { Gain } \\
{[\mathrm{dB}]}\end{array}$ & -37.0 & -22.8 & -24.86 & -23.87 \\
\hline $\begin{array}{c}\text { ONF } \\
{[\mathrm{dBm}]}\end{array}$ & -144.4 & -137.6 & -147.5 & -143.8 \\
\hline $\begin{array}{c}\mathrm{MDIS} \\
{[\mathrm{dBm}]}\end{array}$ & -107.3 & -114.7 & - & - \\
\hline $\begin{array}{c}\text { SFDR } \\
{[\mathrm{dB} / \mathrm{Hz}]}\end{array}$ & 86.0 & 91.4 & $>90$ & $>90$ \\
\hline
\end{tabular}

Fig. 2 (color online) (a) SFDR data from a conventional link and a MiBo link as aquired from experiments and simulations. (b) tabulation of SFDR data, including: input intercept point (IIP3), output intercept point (OIP3), gain (G), output noise floor (ONF), minimum detectable input signal (MDIS), and spur-free dynamic range $\left(\mathrm{SFDR}_{3}\right)$.

Figure 2(a) plots experimental and simulated SFDR. Here, "conventional" denotes a link described by fig. 1(c) where the phase modulator, EDFA, and fiber have been removed from the link. The SFDR plots are generated using equal power 9.89 and $10.11 \mathrm{GHz}$ tones from RF_1 and RF_2, respectively (c.f. fig. 1(c)). The input tone power is swept in steps, and the power produced at the fundamental and intermodulation frequencies are measured using the ESA. A $1 \mathrm{~Hz}$ equivalent noise bandwidth is utilized throughout. The fits are plotted from the noise floor to the intercept point with slope 1 and 3, respectively. Experimental comparison of the MiBo link to the conventional link shows a $14.2 \mathrm{~dB}$ increase in gain and a $7.4 \mathrm{~dB}$ improvement in the minimum detectable input signal (MDIS), while maintaining $\mathrm{SFDR}_{3}$. The difference between simulations and experiment can be explained by ideal RF components and $1 \mathrm{Ohm}$ termination. However, simulated comparions of the MiBo link to the conventional link show a maintained $\mathrm{SFDR}_{3}$. On the other hand, simulations evidence a deterioration in the $\mathrm{SFDR}_{2}$ performance from the conventional to $\mathrm{MiBo}$ case. Experiments to measure $\mathrm{SFDR}_{2}$ are underway. $\mathrm{SFDR}_{2}$ impacts octave spanning RF systems, and will therefore be detailed experimental and numerical studies for such systems in the future. Nevertheless, both experiments and simulations confirm the ability of MiBo to provide net benefit by boosting weak RF signals while maintaining linearity for sub-octave systems. Extending the simulation results to $100 \mathrm{GHz}$ reveal that MiBo links provide more than $30 \mathrm{~dB}$ increase in gain while maintaining $\mathrm{SFDR}_{3}$.

In summary, current experimental and numerical measurements at $10 \mathrm{GHz}$ show a MiBo link boosts RF signals by a relative $14.2 \mathrm{~dB}$ to enable 5 -fold $V_{\pi}$ reduction while achieving an $\mathrm{SFDR}_{3}$ of $91.4 \mathrm{~dB} / \mathrm{Hz}^{2 / 3}$. These results show that the MiBo approach may offer welcomed relief in high frequency applications where RF powers are limited and optical components suffer from linearity.

Acknowledgement: This work was supported by DARPA MTO and SSC Pacific; by ONR; and by Sandia National Laboratories through a graduate research fellowship.

\section{References}

[1] DeVore, P. T. S., et.al., "Enhancing electrooptic modulators using modulation instability," Phys. Status Solidi RRL, 7(8), 566 (2013).

[2] Borlaug, D., et. al., "Demonstration of Vpi Reduction in Electrooptic Modulators Using Modulation Instability," IEEE Photonics Journal, 6(5), (2014).

[3] Agrawal, G. P., "Nonlinear Fiber Optics," Academic Press, Boston, (2007).

[4] Ackerman, Edward I., and C. H. Cox. "Effect of pilot tone-based modulator bias control on external modulation link performance." Proc. Int. Topical Meeting Microw. Photon. Tech. Dig., (2000). 